\begin{tabular}{ccc}
\hline International Journal of Engineering \& Technology, 7 (2.6) (2018) 199-205 \\
SPC & Website: www.sciencepubco.com/index.php/IJET \\
Research Paper & Technology \\
\hline
\end{tabular}

\title{
State Estimation of Power System Embedded with FACTS devices and PMUs
}

\author{
Balaji Venkateswaran $V^{1 *}$, Neeraj Kumar Sharma², Deepali Yadav ${ }^{3}$ \\ ${ }^{1,2,3}$ Dept. of Electrical, Power and Energy, University of Petroleum and Energy Studies, Dehradun, India. \\ *Corresponding author E-mail: b.venkateswaran@ddn.upes.ac.in
}

\begin{abstract}
In recent days, the power system is incorporated with Flexible AC Transmission System (FACTS) devices for compensation of reactive power to maintain the stability of the system. The stability of the system is highly dependent on the state variables which are the outcomes of a state estimator in the power system. To improve the efficiency of a state estimator, high precision measuring devices such as Phasor Measurement Units (PMUs) are installed in the power system. Hence a state estimator embedded with these compensatory devices and PMUs is necessary for estimation of state variables. The present work has been carried out in three steps. Step 1: Considering the cost of PMUs and the availability of the communication network in the particular location, PMUs are optimally placed in the nodes of the system so that all critical measurements are transmuted into redundant ones using differential evolution (DE) algorithm to perform observability analysis. Step 2: A hybrid state estimation is performed by including the mathematical model of FACTS devices and PMUs. Step 3: It is shown that by installing optimal number of PMUs at desired location, multiple bad data detection and identification capability of residual method is considerably improved. Lastly, numerical simulation with standard IEEE 14 bus system, IEEE 118 bus system and a practical 246 bus system of northern region power grid (NRPG) is presented to confirm the effectiveness of the proposed approach in assessing the estimation of the system state variables.
\end{abstract}

Keywords: Observability Analysis; Differential Evolution; State Estimation; Bad data analysis; UPFC; PMU

\section{Introduction}

State Estimation (SE) is an important part in real-time power system monitoring and control. SE approach is used to estimate the state variables (i.e. voltage magnitude and phase angle) based on the measurements from Supervisory Control and Data Acquisition (SCADA) system [1]. SE results are used for subsequent network contingency analysis, security enhancement, and dynamic security applications. The role of SE in Energy Management System (EMS) is discussed in [2]. Usually the measurement set used in Weighted Least Square (WLS) SE algorithm are nodal voltage magnitudes, real and reactive measurements of power flows and power injections. These measurements are transferred to Energy Control Centers (ECC) and are subjected to statistical analysis in order to assess the actual state of power system. Random errors usually exist in measurement due to finite accuracy of the measuring devices and the communication medium. These errors are filtered to eliminate bad data from the measurement set and minimize the random measurement errors. This can be accomplished by using bad data processor. If, after this analysis all state variables of the system can be estimated using the available measurements, a system is said to be observable. Since the work of Fred Schweppe in the late 60s [3-5], exhaustive research has been carried in all the fields of SE starting from observability analysis to bad data processing. Initially, only the line flow measurements are included in the measurement set of SE [6] and later the effects of various mixed measurements are considered in SE approach [7] . Observability analysis is carried out either by topological or numerical approaches. In topological approach, observability is investigated using graph theory based on the type and location of the measurements. In numerical approach, decoupled measurement Jacobian and its gain matrix is used for investigating observability. Several observable islands, each having its own phase angle reference can be formed if we encounter any telecommunication error, meter failure or topology change. These observable islands can be identified by topological approach using incidence matrix by applying gauss elimination method [8]. With the development of optimization algorithms, measuring devices are optimally placed in the power system to make system completely observable [9]. With the invention of GPS and also the development of phasor synchronous measuring devices the observability of the system has been escalated [10]. Considering the cost of PMUs and the geographical constraints for communication, PMUs are optimally placed in a system to make the system completely observable. In a search for optimal location, many algorithms based on linear programming and evolutionary computing have been proposed in [11-15].

On the other hand transmission systems are enduring continuous transformation mainly to increase the power transfer capability of interconnected system due to delay in building of new transmission facilities. The need for more efficient power system management with the advancement of power electronic devices, have given rise to new and powerful semiconductor device such as Flexible AC Transmission System (FACTS) controllers [16]; influencing line power flows to relieve congestion and optimize the overall grid operation [17]. Therefore it is important to integrate FACTS devices model into WLS state estimation algorithm to perform SE. In [18] SE is performed using interior point method by modifying the formulation which incorporates the detailed model of the unified power flow controllers (UPFC) involving the 
equality and inequality constraints of the device that accounts for the limits of the device operation and ratings. The same SE problem involving multiple FACTS devices is formulated as a nonlinear optimization problem with constraints is solved by using predictor - corrector interior point algorithm in [19]. In [20], SE is formulated for the system having UPFC and an injection from high voltage dc (HVDC) side. In this formulation the net injection measured from HVDC side is assumed to be error free. Since all conventional measurements which make the system observable are with finite accuracy, bad data analysis is required in the process of state estimation. Earlier, bad data detection in the measurement set can be achieved using chi - square test. However, it failed to detect multiple bad data in the measurement set. Later, the introduction of high precision PMUs into the system, have considerably reduced the chance of erroneous measurement thereby improved the performance of SE [21]

In this paper an extended weighted least square state estimator (WLS-SE) algorithm has been developed keeping the operating constraints of the controller within operating bounds. UPFC controller modelled in [22] is modified and integrated into the WLSSE formulation. To improve the accuracy of SE in stages of observability and bad data detection, PMU measurements are included along with conventional measurements, where PMUs are optimally placed in the system using DE algorithm. Also, multiple bad data detection and identification is performed using largest normalized residual method.

\section{State Estimation Formulation}

State estimation procedure formulated in this paper assess the best estimates of the system state variables $x^{k}$ that fits available measurement set $z$, collected from SCADA and PMU measurements: $z=\left[z^{S C A D A} z^{P M U}\right]^{T}$ the superscript $T$ indicates transposition.

$z=h\left(x^{k}\right)+\varepsilon$

where $x^{k}$ is a vector of nodal voltages, branch currents, and branch power flow and also power injections by generators and FACTS devices. $h\left(x^{k}\right)=\left[h^{S C A D A} h^{P M U}\right]:$ refers the estimated measurements through mathematical models. $\varepsilon$ : represents the imperfections of real time measuring devices. WLS state estimation provides solution to the normal equation given by (2), iteratively [1] and newly corrected vector is given by $x^{k+1}=x^{k}+\Delta x^{k}$. The optimal state vector $x^{k}$ is found if $\left|\Delta x^{k}\right| \leq \mathrm{E}$ is fulfilled.

$\Delta x^{k}=H^{T}\left(x^{k}\right) R^{-1}\left[z-h\left(x^{k}\right)\right] G\left(x^{k}\right)^{-1}$

The error covariance matrix and Jacobian matrix are given by (3) and (4), respectively.

$R=\left[\begin{array}{cc}{\left[R^{S C A D A}\right]} & 0 \\ 0 & {\left[R^{P M U}\right]}\end{array}\right]$

$H\left(x^{k}\right)=\left[\begin{array}{c}H^{S C A D A} \\ H^{P M U}\end{array}\right]=\left[\begin{array}{cc}\frac{\partial P_{i n j}}{\partial \theta^{k}} & \frac{\partial P_{i n j}}{\partial V^{k}} \\ \frac{\partial P_{\text {flow }}}{\partial \theta^{k}} & \frac{\partial P_{\text {flow }}}{\partial V^{k}} \\ \frac{\partial Q_{i n j}}{\partial \theta^{k}} & \frac{\partial Q_{\text {inj }}}{\partial V^{k}} \\ \frac{\partial Q_{\text {flow }}}{\partial \theta^{k}} & \frac{\partial Q_{\text {flow }}}{\partial V^{k}} \\ \frac{\partial V_{\text {mag }}}{\partial \theta^{k}} & \frac{\partial V_{\text {mag }}}{\partial V^{k}} \\ \frac{\partial \theta^{P M U}}{\partial \theta^{k}} & \frac{\partial \theta^{P M U}}{\partial V^{k}} \\ \frac{\partial V^{P M U}}{\partial \theta^{k}} & \frac{\partial V^{P M U}}{\partial V^{k}} \\ \frac{\partial I_{\text {ral }}}{\partial \theta^{k}} & \frac{\partial I_{\text {real }}^{P M U}}{\partial V^{k}} \\ \frac{\partial I_{\text {imag }}^{P^{k}}}{\partial \theta^{k}} & \frac{\partial I_{\text {imag }}^{P M U}}{\partial \theta^{k}}\end{array}\right]$ where the covariance matrices is given by the set of variance of measurements as their diagonal elements:

$\left[R^{S C A D A}\right]=\operatorname{diag}\left\{\sigma_{P_{i n j}}^{2} \sigma_{Q_{i n j}}^{2} \sigma_{P_{\text {flow }}}^{2} \sigma_{Q_{\text {flow }}}^{2} \sigma_{V}^{2}\right\}$ and

$\left[R^{P M U}\right]=\operatorname{diag}\left\{\sigma_{V_{\text {mag }}^{P M U}}^{2} \sigma_{\theta^{P M U}}^{2} \sigma_{I_{\text {real }}^{P M U}}^{2} \sigma_{I_{\text {imag }}^{P M U}}^{2}\right\}$

\section{Mathematical modeling of SCADA and PMU Measurements}

The mathematical function for SCADA measurements and UPFC are detailed in [1] and [22], respectively. On the other hand, the mathematical function of PMU measurements relating to the conventional measurement state variables and FACTS controller must be modelled in order to be considered into state estimation algorithm. The synchronized measurements of current phasors are considered in rectangular form and its nonlinear equations relating branch current phasors are derived below:

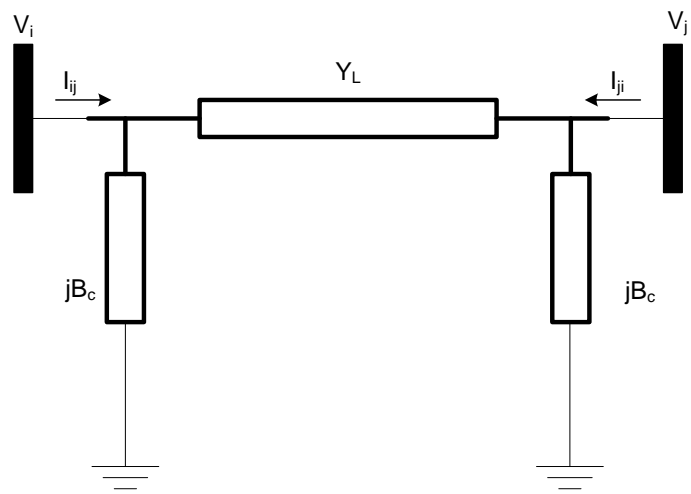

Fig. 1: Transmission Line $\pi$ model

\subsection{Modeling of branch current and voltage phasor measurements}

Considering the PMU is installed at bus $i$, the rectangular form of the phasors associated with the branch current flowing from node $i$ to node $j$ through $\pi$ transmission line shown in Figure 1 are given by

$I_{i j}=V_{i} Y_{i i}+Y_{i j} V_{j}$

where the admittance of the transmission line is: $Y_{L}=Y_{i j}=g_{i j}+j b_{i j}$ The real and imaginary part of the branch current phasor is expressed as:

$I_{\text {real }}^{P M U}=V_{i}^{k}\left(g_{i i} \cos \theta_{i}^{k}-b_{i i} \sin \theta_{i}^{k}\right)+\left(g_{i j} \cos \theta_{j}^{k}-b_{i j} \sin \theta_{j}^{k}\right) V_{j}^{k}$

$I_{\text {imag }}^{P M U}=V_{i}^{k}\left(g_{i i} \sin \theta_{i}^{k}-b_{i i} \cos \theta_{i}^{k}\right)+\left(g_{i j} \sin \theta_{j}^{k}-b_{i j} \cos \theta_{j}^{k}\right) V_{j}^{k}$

where the complex nodal admittance are $Y_{i i}=y_{i j}+j B_{c}=g_{i i}+j b_{i i}$ and $Y_{i j}=-y_{i j}=g_{i j}+j b_{i j}$. The Jacobian matrix elements of branch current phasors and voltage phasors in equation (3) are given by equations (8) - (11) and (12) - (15) respectively.

$\frac{\partial I_{\text {real }}^{P M U}}{\partial \theta^{k}} \rightarrow\left\{\begin{array}{l}\frac{\partial I_{\text {real }}^{P M U}}{\partial \theta_{i}^{k}}=-V_{i}^{k}\left(g_{i i} \sin \theta_{i}^{k}+b_{i i} \cos \theta_{i}^{k}\right) \\ \frac{\partial I_{\text {real }}^{P M U}}{\partial \theta_{j}^{k}}=-V_{j}^{k}\left(g_{i j} \sin \theta_{j}^{k}+b_{i j} \cos \theta_{j}^{k}\right)\end{array}\right.$ 


$$
\begin{aligned}
& \frac{\partial I_{\text {real }}^{P M U}}{\partial V^{k}} \rightarrow\left\{\begin{array}{l}
\frac{\partial I_{\text {real }}^{P M U}}{\partial V_{i}^{k}}=\left(g_{i i} \cos \theta_{i}^{k}-b_{i i} \sin \theta_{i}^{k}\right) \\
\frac{\partial I_{r e a l}^{P M U}}{\partial V_{j}^{k}}=\left(g_{i j} \cos \theta_{j}^{k}-b_{i j} \sin \theta_{j}^{k}\right)
\end{array}\right. \\
& \frac{\partial I_{\text {imag }}^{P M U}}{\partial \theta^{k}} \rightarrow\left\{\begin{array}{l}
\frac{\partial I_{\text {imag }}^{P M U}}{\partial \theta_{i}^{k}}=V_{i}^{k}\left(g_{i i} \cos \theta_{i}^{k}-b_{i i} \sin \theta_{i}^{k}\right) \\
\frac{\partial I_{\text {imag }}^{P M U}}{\partial \theta_{j}^{k}}=V_{j}^{k}\left(g_{i j} \cos \theta_{j}^{k}-b_{i j} \sin \theta_{j}^{k}\right)
\end{array}\right. \\
& \frac{\partial I_{i m a g}^{P M U}}{\partial V^{k}} \rightarrow\left\{\begin{array}{l}
\frac{\partial I_{i m a g}^{P M U}}{\partial V_{i}^{k}}=\left(g_{i i} \sin \theta_{i}^{k}+b_{i i} \cos \theta_{i}^{k}\right) \\
\frac{\partial I_{i m a g}^{P M U}}{\partial V_{j}^{k}}=\left(g_{i j} \sin \theta_{j}^{k}+b_{i j} \cos \theta_{j}^{k}\right)
\end{array}\right.
\end{aligned}
$$

The equations for the complex current phasor $I_{j i}$ can be obtained by replacing the subscripts in equations (8)-(11)

$$
\begin{aligned}
& \frac{\partial \theta^{P M U}}{\partial \theta^{k}}= \begin{cases}1 & , \quad i=j \\
0, & i \neq j\end{cases} \\
& \frac{\partial \theta^{P M U}}{\partial V^{k}}=0 \quad, \forall i \\
& \frac{\partial V^{P M U}}{\partial \theta^{k}}=0 \quad, \forall i \\
& \frac{\partial V^{P M U}}{\partial V^{k}}= \begin{cases}1 \quad, \quad i=j \\
0 \quad, \quad i \neq j\end{cases}
\end{aligned}
$$

\subsection{Modeling of branch current phasor measurement of UPFC}

The UPFC is a combination of static synchronous compensator (STATCOM) and static series compensator (SSSC), which are coupled together via a common de link, to allow bidirectional flow of real power between series output terminals of the SSSC and the shunt output terminals of the STATCOM. A simplified schematic representation of the UPFC is given in figure 2 [22]

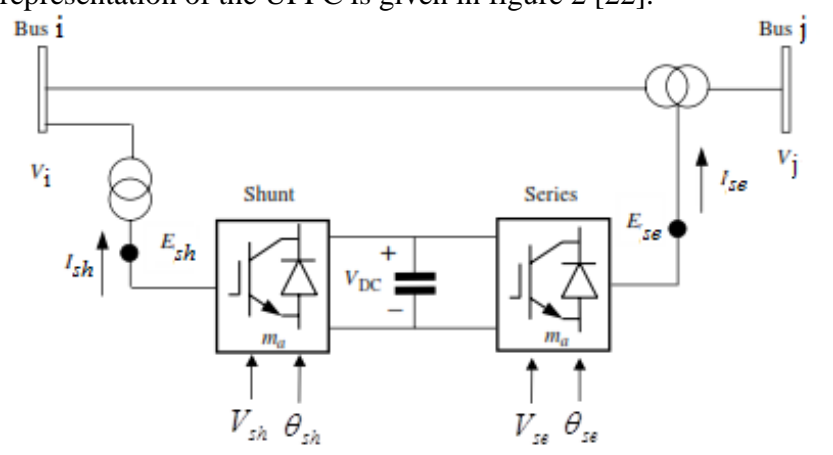

Fig. 2: A simplified schematic of UPFC

The basic function of shunt converter in figure 2 is to supply or absorb the real power demanded by the series converter at the dc link to provide series active compensation. In addition to real power supply of shunt converter, it can also generate or absorb controllable reactive power thereby providing shunt reactive compensation. The equivalent circuit of UPFC is modeled with two voltage source converters (VSCs) sharing a common capacitor on their $\mathrm{dc}$ side as shown in figure 3 along with a unified control system. The output voltage of series converter is added to the nodal voltage, say at bus $i$, to boost the nodal voltage at bus $j$. The voltage magnitude of the output voltage $V_{\text {se }}$ provides voltage regulation, and the phase angle $\theta_{\text {se }}$ determines the mode of power flow control.

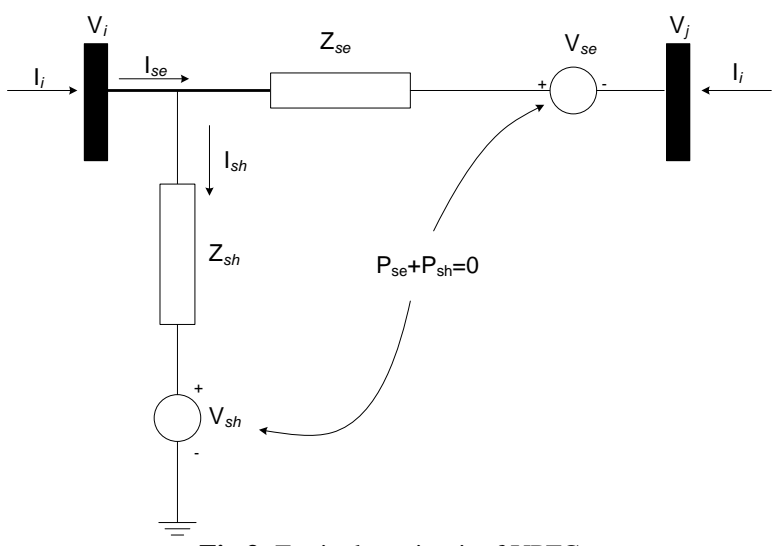

Fig.3: Equivalent circuit of UPFC

The branch current phasors are derived by considering complex voltages and the nodal voltage are given by the equation below:

$V_{x}^{k}=V_{x}\left(\cos \theta_{x}+j \sin \theta_{x}\right) \quad \forall x=s e, s h, i, j$

which is based on the equivalent circuit in figure 3 and assuming the UPFC is connected to bus $i$ of figure 4; the following transfer admittance equation can be written by:

$\left[\begin{array}{c}I_{i} \\ I_{j}\end{array}\right]=\left[\begin{array}{cccc}\left(Y_{L}+j B_{c}+Y_{s e}+Y_{s h}\right) & -\left(Y_{L}+Y_{s e}\right) & -\left(Y_{L}+Y_{s e}\right) & -Y_{s h} \\ -\left(Y_{L}+Y_{s e}\right) & \left(Y_{L}+j B_{c}+Y_{s e}\right. & \left(Y_{L}+j B_{c}+Y_{s e}\right. & 0\end{array}\right]\left[\begin{array}{c}V_{i} \\ V_{j} \\ V_{s e} \\ V_{s h}\end{array}\right]$

Where

$Y_{s e}=\frac{1}{Z_{s e}}=\frac{1}{R_{s e}+j X_{s e}} ; Y_{s h}=\frac{1}{Z_{s h}}=\frac{1}{R_{s h}+j X_{s h}} ; Y_{L}=\frac{1}{Z_{L}}$

Let $\left(G_{i i}+j B_{i i}\right)=Y_{i i}=\left(Y_{L}+j B_{c}+Y_{s e}+Y_{s h}\right)$,

$\left(G_{j j}+j B_{j j}\right)=Y_{j j}=\left(Y_{L}+j B_{c}+Y_{s e}\right)$,

$\left(G_{i j}+j B_{i j}\right)=Y_{i j}=Y_{j i}=-\left(Y_{L}+Y_{s e}\right)$

and $\left(G_{s h}+j B_{s h}\right)=-Y_{s h}$

By substituting (17) and (18) in (16), the branch current phasors at the terminals of UPFC can be obtained as:

$$
\begin{aligned}
& I_{i}=\left(G_{i i}+j B_{i i}\right) V_{i}+\left(G_{i j}+j B_{i j}\right) V_{j}+\left(G_{i j}+j B_{i j}\right) V_{s e}+\left(G_{s h}+j B_{s h}\right) V_{s h} \\
& I_{j}=\left(G_{i j}+j B_{i j}\right) V_{i}+\left(G_{i i}+j B_{i i}\right) V_{j}+\left(G_{i i}+j B_{i i}\right) V_{s e}
\end{aligned}
$$

The power injected at bus $i$ and $j$ is the sum of normal power injected into the bus and the power injection due to UPFC as shown in figure 4 . Thus the power injections at bus $i$ and $j$ is given by: 


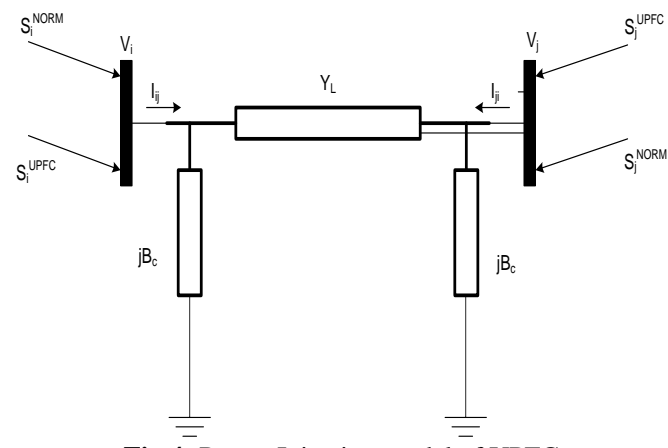

Fig.4: Power Injection model of UPFC

$P_{i n j, i}=P_{n o r m, i}+\Delta P_{i n j, i}$

$Q_{i n j, i}=Q_{n o r m, i}+\Delta Q_{i n j, i}$

where $P_{n o r m, i}, Q_{\text {norm }, i}$ are the normal active and reactive power

injections at bus $i$. Using equation (19), we can derive the active and reactive power injections by UPFC at bus $i$ as:

$P_{i n j, i}=V_{i}^{k} \sum_{j=1}^{N}\left\{V_{j}^{k}\left(g_{i j} \cos \theta_{i j}+b_{i j} \sin \theta_{i j}\right)\right\}+V_{i}^{k}\left\{V_{i}^{k}\left(G_{i i} \cos \theta_{i}^{k}-B_{i i} \sin \theta_{i}^{k}\right)+V_{j}^{k}\left(G_{i j} \cos \theta_{j}^{k}-B_{i j} \sin \theta_{j}^{k}\right)\right.$

$\left.+V_{s e}^{k}\left(G_{i j} \cos \theta_{s e}^{k}-B_{i j} \sin \theta_{s e}^{k}\right)+V_{s h}^{k}\left(G_{s h} \cos \theta_{s h}^{k}-B_{s h} \sin \theta_{s h}^{k}\right)\right\}$

$Q_{i n j, i}=V_{i}^{k} \sum_{j=1}^{N}\left\{V_{j}^{k}\left(g_{i j} \sin \theta_{i j}-b_{i j} \cos \theta_{i j}\right)\right\}+V_{i}^{k}\left\{V_{i}^{k}\left(G_{i i} \sin \theta_{i}^{k}+B_{i i} \cos \theta_{i}^{k}\right)+V_{j}^{k}\left(G_{i j} \sin \theta_{j}^{k}+B_{i j} \cos \theta_{j}^{k}\right)\right.$

$\left.+V_{s e}^{k}\left(G_{i j} \sin \theta_{s e}^{k}+B_{i j} \cos \theta_{s e}^{k}\right)+V_{s h}^{k}\left(G_{s h} \sin \theta_{s h}^{k}+B_{s h} \cos \theta_{s h}^{k}\right)\right\}$

$\frac{\partial P_{i n j, i}}{\partial \theta_{i}^{k}}=\sum_{j=1}^{N} V_{i}^{k} V_{j}^{k}\left(-g_{i j} \sin \theta_{i j}+b_{i j} \cos \theta_{i j}\right)-\left(\left(V_{i}^{k}\right)^{2}\left(G_{i i} \sin \theta_{i}^{k}+B_{i i}\left(1+\cos \theta_{i}^{k}\right)\right)\right)$

$\frac{\partial P_{i n j, i}}{\partial \theta_{j}^{k}}=V_{i}^{k} V_{j}^{k}\left(g_{i j} \sin \theta_{i j}-b_{i j} \cos \theta_{i j}\right)-V_{i}^{k} V_{j}^{k}\left(G_{i j} \sin \theta_{j}^{k}+B_{i j} \cos \theta_{j}^{k}\right)$

$\frac{\partial P_{i n j, i}}{\partial V_{i}^{k}}=\sum_{j=1}^{N} V_{j}^{k}\left(g_{i j} \cos \theta_{i j}+b_{i j} \sin \theta_{i j}\right)+V_{i}^{k} G_{i i}+2 V_{i}^{k}\left(G_{i i} \cos \theta_{i}^{k}-B_{i i} \sin \theta_{i}^{k}\right)$

$\frac{\partial P_{i n j, i}}{\partial V_{j}^{k}}=V_{i}^{k}\left(g_{i j} \cos \theta_{i j}+b_{i j} \sin \theta_{i j}\right)+V_{i}^{k}\left(G_{i j} \cos \theta_{i}^{k}-B_{i j} \sin \theta_{i}^{k}\right)$

$\frac{\partial Q_{i n j, i}}{\partial \theta_{i}^{k}}=\sum_{j=1}^{N} V_{i}^{k} V_{j}^{k}\left(g_{i j} \cos \theta_{i j}+b_{i j} \sin \theta_{i j}\right)+\left(V_{i}^{k}\right)^{2}\left\{G_{i i}\left(\cos \theta_{i}^{k}-1\right)-B_{i i} \sin \theta_{i}^{k}\right\}$

$\frac{\partial Q_{i n j, i}}{\partial \theta_{j}^{k}}=V_{i}^{k} V_{j}^{k}\left(-g_{i j} \cos \theta_{i j}-b_{i j} \sin \theta_{i j}\right)-V_{i}^{k} V_{j}^{k}\left(G_{i j} \cos \theta_{j}^{k}-B_{i j} \sin \theta_{j}^{k}\right)$

$\frac{\partial Q_{i n j, i}}{\partial V_{i}^{k}}=\sum_{j=1}^{N} V_{j}^{k}\left(g_{i j} \sin \theta_{i j}-b_{i j} \cos \theta_{i j}\right)-V_{i}^{k} B_{i i}+2 V_{i}^{k}\left(G_{i i} \sin \theta_{i}^{k}+B_{i i} \cos \theta_{i}^{k}\right)$

$\frac{\partial Q_{i n j, i}}{\partial V_{j}^{k}}=V_{i}^{k}\left(g_{i j} \sin \theta_{i j}-b_{i j} \cos \theta_{i j}\right)+V_{i}^{k}\left(G_{i j} \sin \theta_{i}^{k}+B_{i j} \cos \theta_{i}^{k}\right)$

\subsection{Observability analysis and bad data analysis}

In this paper observability analysis is performed by placing the PMUs at optimal location to have a completely observable system with minimum number of PMUs. The objective function of this optimization problem is formulated as equation (35). This optimization problem is solved by using DE algorithm as implemented in [14].

$$
\begin{aligned}
& \Delta P_{i n j, i}=V_{i}^{k}\left\{V_{i}^{k}\left(G_{i i} \cos \theta_{i}^{k}-B_{i i} \sin \theta_{i}^{k}\right)+V_{j}^{k}\left(G_{i j} \cos \theta_{j}^{k}-B_{i j} \sin \theta_{j}^{k}\right)\right. \\
& \left.+V_{s e}^{k}\left(G_{i j} \cos \theta_{s e}^{k}-B_{i j} \sin \theta_{s e}^{k}\right)+V_{s h}^{k}\left(G_{s h} \cos \theta_{s h}^{k}-B_{s h} \sin \theta_{s h}^{k}\right)\right\} \\
& \Delta Q_{i n j, i}=V_{i}^{k}\left\{V_{i}^{k}\left(G_{i i} \sin \theta_{i}^{k}+B_{i i} \cos \theta_{i}^{k}\right)+V_{j}^{k}\left(G_{i j} \sin \theta_{j}^{k}+B_{i j} \cos \theta_{j}^{k}\right)\right. \\
& \left.+V_{s e}^{k}\left(G_{i j} \sin \theta_{s e}^{k}+B_{i j} \cos \theta_{s e}^{k}\right)+V_{s h}^{k}\left(G_{s h} \sin \theta_{s h}^{k}+B_{s h} \cos \theta_{s h}^{k}\right)\right\}
\end{aligned}
$$

Substituting the equations for normal power injections detailed in [1] in equations (21) - (24) will give the total power injections at bus $i$ as shown in equations (25) and (26).

The elements of Jacobian matrix in equation (4) related to active reactive power injections at bus $i$ are derived using equations (26) which are given by equations (27) - (34). The active and reactive power injection at bus $j$ can be attained just by exchanging the subscripts.

(ach

$$
\text { proce }
$$
urement set. Conventionally, detection of bad data is accompliontention of bad data is accomized residual test is employed to identify single bad data measurements and also for multiple bad data [1]. The normalized residuals are given by equation (36).

$$
r_{i}^{N}=\frac{\left|r_{i}\right|}{\sqrt{\Omega_{i i}}} \quad i=1, \ldots, m
$$

where $r_{i}=z_{i}-h\left(x^{k}\right)$ and $\Omega_{i i}$ is the diagonal elements of the residual covariance matrix given by equation (37) which is calculated with modified Jacobian matrix. The complete procedure of state estimation proposed in this paper is charted in flowchart as shown in figure 5.

$$
\Omega=R-H\left(x^{k}\right) G^{-1} H\left(x^{k}\right)^{T}
$$

\section{Study Cases}

The performance of the proposed SE method is reported in this section using two practical power system networks: (1) IEEE $14-$ bus system and (2) IEEE 118 - bus system. Studies were carried out for three cases namely (i) performing SE with conventional measurements and UPFC, (ii) performing SE with optimally placed PMUs and UPFC and (iii) performing SE with conventional measurements, optimally placed PMUs and UPFC. For all the cases, the UPFC's coupling transformer has the same impedances: $Z_{s e}=Z_{s h}=0.05+j 0.1 p u$ The input measurement set provided 
to SE is obtained from Newton Raphson power flow including UPFC [24]. The random errors added to the measurements with standard deviation values are shown in Table 1 [25]. The tolerance criteria considered for all cases is $\varepsilon=1 e-6$.

Table 1: Measurement Standard Deviation

\begin{tabular}{ccccc}
\hline $\begin{array}{c}\text { Measurement } \\
\text { Type }\end{array}$ & $\begin{array}{c}\text { Injection } \\
\text { (p.u.) }\end{array}$ & $\begin{array}{c}\text { Flow } \\
\text { (p.u.) }\end{array}$ & $\begin{array}{c}\text { Voltage } \\
\text { Magnitude } \\
\text { (p.u.) }\end{array}$ & $\begin{array}{c}\text { Angle } \\
\text { (rad) }\end{array}$ \\
\hline $\begin{array}{c}\text { SCADA meas- } \\
\text { urements } \\
\text { PMU meas- } \\
\text { urements }\end{array}$ & 0.01 & 0.008 & 0.004 & - \\
\hline
\end{tabular}

\subsection{IEEE 14-bus system}

The IEEE 14 - bus system has been modified with one UPFC in series with the transmission line connected at the bus $6-12$ for all cases along with conventional measurements. For the second case, the IEEE 14 - bus system is modified with PMUs connected at the buses 2, 6, 9 and 12 along with UPFC. For the third case, the IEEE 14- bus system is modified with PMUs connected at the buses 3, 6, 9 along with UPFC. Considering the cost of PMU as a constraint, the optimal locations for case ii and case iii are ob- tained by performing optimization algorithm using DE [14]. The set of conventional measurements provided by SCADA system composed of 12 pairs of power flow measurements, 8 pairs of power injection measurements and one voltage magnitude measurement with four power flow measurements related to UPFC for all three cases. In addition, one pseudo measurement is included to take the active power balance constraint at dc link into account. The PMUs placed at the above mentioned buses provide both nodal voltage and branch current phasor measurements with four nodal voltage phasor measurements and twenty branch current phasor measurements for case ii and three nodal voltage phasor measurements and ten branch current phasor measurements for case iii. The phase angle measurement of the slack bus is not considered in SE algorithm. The initial condition of voltage sources representing UPFC's series and shunt converters are $V_{s e}=0.50 \angle 0^{\circ}$ and $V_{s h}=1.0 \angle 0^{\circ}$ respectively. The results of $\mathrm{SE}$ algorithm for all the cases are shown in Table 2. The error of voltage magnitude corresponding to all the three cases is shown in figure 6 . The degree of freedom yields the relation between the available measurements and the state variables of the system to be estimated. The degree of freedom for all the three cases and the corresponding test threshold is shown in Table 3.

Table 2: Voltage phasors of all the three cases

\begin{tabular}{|c|c|c|c|c|c|c|c|c|}
\hline \multirow{2}{*}{ Bus no } & \multicolumn{2}{|c|}{ True Measurements } & \multicolumn{2}{c|}{ Case i } & \multicolumn{2}{c|}{ Case ii } & \multicolumn{2}{c|}{ Case iii } \\
\cline { 2 - 9 } & $\mathbf{V}(\mathbf{p u})$ & $\boldsymbol{\theta}(\mathbf{p u})$ & $\mathbf{V}(\mathbf{p u})$ & $\boldsymbol{\theta}(\mathbf{p u})$ & $\mathbf{V}(\mathbf{p u})$ & $\boldsymbol{\theta}(\mathbf{p u})$ & $\mathbf{V}(\mathbf{p u})$ & $\boldsymbol{\theta}(\mathbf{p u})$ \\
\hline 1 & 1.0600 & 0 & 1.0600 & 0 & 1.0600 & 0 & 1.0665 & 0 \\
\hline 2 & 1.0450 & -0.0871 & 1.0430 & -0.0934 & 1.0450 & -0.0872 & 1.0506 & -0.0771 \\
\hline 3 & 1.0100 & -0.2225 & 1.0200 & -0.1315 & 1.0103 & -0.2245 & 1.0478 & -0.2252 \\
\hline 4 & 1.0132 & -0.1788 & 1.0108 & -0.1620 & 1.0135 & -0.1768 & 1.0332 & -0.1778 \\
\hline 5 & 1.0166 & -0.1529 & 1.0100 & -0.2473 & 1.0166 & -0.1499 & 1.0498 & -0.1532 \\
\hline 6 & 1.0700 & -0.2521 & 1.0101 & -0.1931 & 1.0701 & -0.2531 & 1.1143 & -0.2561 \\
\hline 7 & 1.0457 & -0.2310 & 1.0023 & -0.2245 & 1.0457 & -0.2320 & 1.0394 & -0.2510 \\
\hline 8 & 1.0800 & -0.2310 & 1.0100 & -0.2062 & 1.0805 & -0.2370 & 1.0492 & -0.2210 \\
\hline 9 & 1.0305 & -0.2587 & 1.0402 & -0.2452 & 1.0303 & -0.2517 & 1.0560 & -0.2887 \\
\hline 10 & 1.0299 & -0.2624 & 1.0231 & -0.2731 & 1.0300 & -0.2664 & 1.0388 & -0.2324 \\
\hline 11 & 1.0461 & -0.2593 & 1.0820 & -0.2452 & 1.0468 & -0.2553 & 1.0411 & -0.2693 \\
\hline 12 & 1.0533 & -0.2670 & 1.0503 & -0.2634 & 1.0532 & -0.2680 & 1.1202 & -0.2470 \\
\hline 13 & 1.0466 & -0.2676 & 1.0710 & -0.2634 & 1.0468 & -0.2636 & 1.0870 & -0.2876 \\
\hline 14 & 1.0193 & -0.2805 & 1.0330 & -0.2787 & 1.0191 & -0.2800 & 1.0642 & -0.2105 \\
\hline
\end{tabular}

urements decreases the error on the estimated voltage phasors of

The bad data detection and identification is performed using largest normalized method with $c=3$. The noise introduced into the measurement data $P_{4-7} P_{2} P_{10-11}$ and $P_{4}$ is successfully identified by largest normalized method.

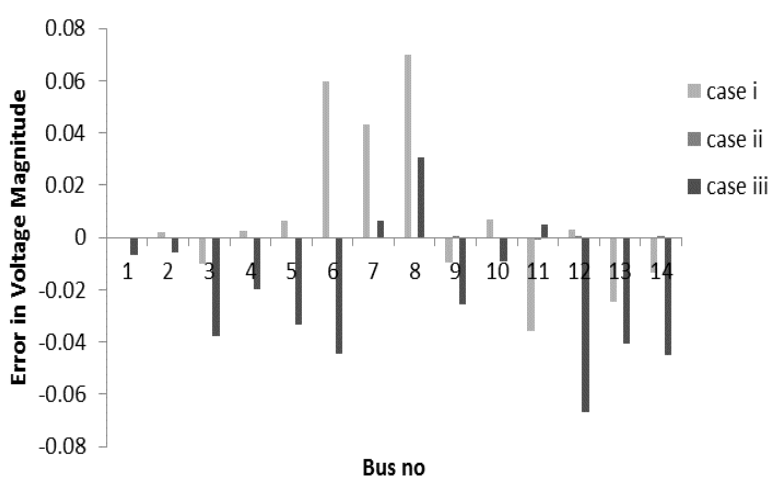

Fig. 5: Errors of Voltage Magnitude for all the three cases

Even though the degree of freedom for second case is very low in table 3, the SE algorithm gives accurate results, since the PMUs are assumed to be of high precision. The accuracy of each case is illustrated in Table 4 in terms of mean square error (MSE), maximum voltage magnitude error (MVME) and maximum voltage angle error (MVAE). This table clearly shows that introduction of only PMUs into the system increases the accuracy of SE and introduction of optimal number of PMUs with conventional meas- the system.

Table 3: Degree of Freedom

\begin{tabular}{ccc}
\hline Cases & Degree of freedom & Test threshold $\aleph^{2}$ \\
\hline Case i & 27 & 46.9629 \\
Case ii & 10 & 23.2093 \\
Case iii & 40 & 63.6907 \\
\hline
\end{tabular}

Table 4: Comparison of Errors for all cases

\begin{tabular}{cccc}
\hline & Case i & Case ii & Case iii \\
\hline MSE & 0.00091 & $7.64 \mathrm{E}-08$ & 0.0010596 \\
MVME & 0.07 & 0.0002 & 0.0308 \\
MVAE & 0.0944 & 0.006 & 0.03 \\
\hline
\end{tabular}

\subsection{IEEE 118-bus system}

The IEEE 118 - bus system has been modified with one UPFC in series with the transmission line connected at the bus $30-38$ and 81- 68 for all cases along with conventional measurements. For the second case, the IEEE 118 - bus system is modified with PMUs connected at the buses as mentioned in [14] along with UPFC. For the third case, the IEEE 14- bus system is modified with PMUs connected at the buses as mentioned in [14] along with UPFC. The set of conventional measurements provided by SCADA system composed of 111 pairs of power flow measurements, 39 pairs of power injection measurements and one voltage magnitude measurement with four power flow measurements related to UPFC for all three cases. In addition, one pseudo measurement is included to take the active power balance constraint at 
dc link into account. The PMUs placed at the above mentioned buses provide both nodal voltage and branch current phasor measurements. The phase angle measurement of the slack bus is not considered in SE algorithm. The initial condition of voltage sources representing UPFC's series and shunt converters are $V_{s e}=0.070 \angle 81.80^{\circ}$ and $V_{s h}=1.0 \angle 0^{\circ}$ respectively. The degree of freedom yields the relation between the available measurements and the state variables of the system to be estimated. The degree of freedom for all the three cases and the corresponding test threshold is shown in Table 5.

Table 5: Degree of Freedom

\begin{tabular}{ccc}
\hline Cases & Degree of freedom & Test threshold $\boldsymbol{\aleph}^{2}$ \\
\hline Case i & 186 & 233.7862 \\
Case ii & 14 & 29.1412 \\
Case iii & 318 & 379.5916 \\
\hline
\end{tabular}

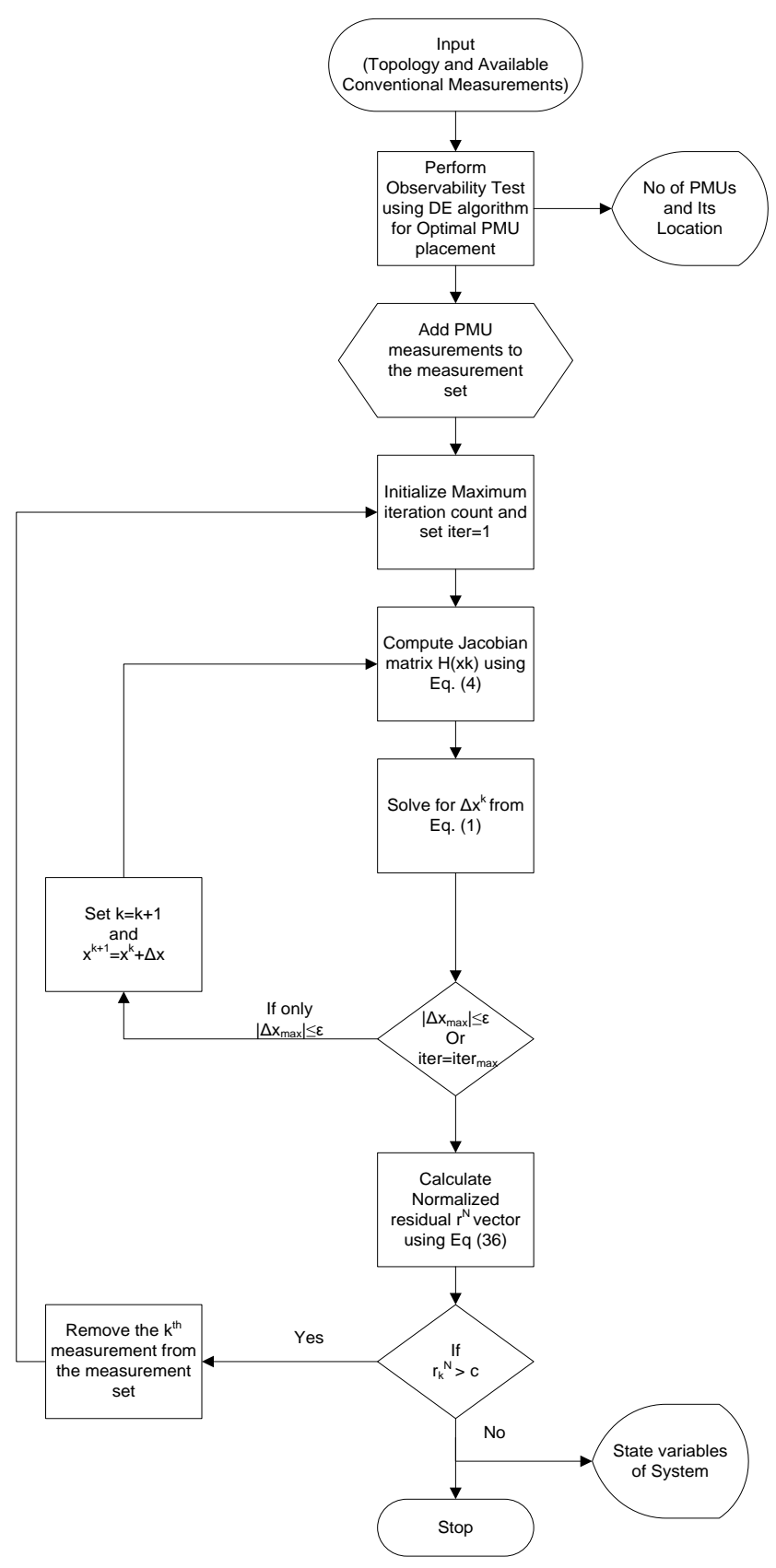

Fig. 6: Proposed State Estimation Algorithm
Table 6: Comparison of Errors for all cases

\begin{tabular}{cccc}
\hline & Case i & Case ii & Case iii \\
\hline MSE & 0.00901 & $7.64 \mathrm{E}-08$ & 0.1996 \\
MVME & 0.0709 & 0.0286 & 0.398 \\
MVAE & 0.0914 & 0.2076 & 0.331 \\
\hline
\end{tabular}

A comparison of errors for each case is illustrated in Table 6 in terms of mean square error (MSE), maximum voltage magnitude error (MVME) and maximum voltage angle error (MVAE). This table clearly shows that introduction of only PMUs into the system increases the accuracy of SE and introduction of optimal number of PMUs with conventional measurements decreases the error on the estimated voltage phasors of the system. The bad data detection and identification is performed using largest normalized method with $c=3$ by introducing the noise for $Q_{7-12} P_{50} P_{93-92}$ and $P_{11}$ is successfully identified by largest normalized method.

\section{Conclusion}

A weighted least square formulation appropriate for the estimation of state of a power system containing FACTS device through measurements provided by SCADA system and PMUs has been proposed in this paper with optimal placement of PMU using DE. Estimation of two practical transmission networks has been performed in order to illustrate the proposed approach as well as the optimal location of PMUs on the accuracy of state estimation. It has been shown that with the optimal number of PMUs in the system along with conventional measurements, increases the measurement redundancy that permits to detection and identification bad data with increase in accuracy.

\section{References}

[1] Abur A, Expósito AG. Power system state estimation: theory and implementation. Power 2004:327.

[2] Dy Liacco TE. The role and implementation of state estimation in an energy management system. Int J Electr Power Energy Syst 1990;12:75-9. doi:10.1016/0142-0615(90)90002-S.

[3] Schweppe F, Wildes J. Power System Static-State Estimation, Part I: Exact Model. IEEE Trans Power Appar Syst 1970;PAS89:120-5. doi:10.1109/TPAS.1970.292678.

[4] Schweppe F, Rom D. Power System Static-State Estimation, Part II: Approximate Model. IEEE Trans Power Appar Syst 1970;PAS-89:125-30. doi:10.1109/TPAS.1970.292679.

[5] Schweppe F. Power System Static-State Estimation, Part III: Implementation. IEEE Trans Power Appar Syst 1970;PAS-89:130 5. doi:10.1109/TPAS.1970.292680.

[6] Hubbi W, Shirazi F. A line flow only power system state estimator. Electr Power Syst Res 1990;19:229-37. doi:10.1016/03787796(90)90036-3.

[7] Ding Junce, Cai Zexiang. Mixed Measurements State Estimation Based on Wide-Area Measurement System and Analysis. 2005 IEEE/PES Transm. Distrib. Conf. Expo. Asia Pacific, IEEE; 2005, p. 1-5. doi:10.1109/TDC.2005.1546795.

[8] Moon YH, Park YM, Lee KJ. Observable island identification for state estimation using incidence matrix. Automatica 1988;24:71-5. doi:10.1016/0005-1098(88)90008-8.

[9] Clements KA. Observability methods and optimal meter placement. Int J Electr Power Energy Syst 1990;12:88-93. doi:10.1016/0142-0615(90)90004-U.

[10] Boisen MB. Power System Observability With Minimal Phasor Measurement Placement 1993;8:707-15.

[11] Gou B. Optimal Placement of PMUs by Integer Linear Programming 2008;23:1525-6.

[12] Hajian M, Ranjbar AM, Amraee T, Shirani AR. Optimal Placement of Phasor Measurement Units : Particle Swarm Optimization Approach n.d.

[13] Aminifar F, Fotuhi-firuzabad M, Safdarian A. Optimal PMU Placement Based on Probabilistic Cost/Bene fi $t$ Analysis 2012;28:1-2.

[14] Venkateswaran VB, Kala VSC. Observability analysis and optimal placement of PMU using Differential Evolution algorithm. 
2012 Int. Conf. Emerg. Trends Electr. Eng. Energy Manag., IEEE; 2012, p. 205-9. doi:10.1109/ICETEEEM.2012.6494461.

[15] Chakrabarti S, Kyriakides E. Optimal placement of phasor measurement units for power system observability. IEEE Trans Power Syst 2008;23:1433-40. doi:10.1109/TPWRS.2008.922621.

[16] Narain G. Hingorani; Laszlo Gyugyi. Understanding FACTS Concepts and Technology. vol. XXXIII. IEEE Press Marketing; 2000. doi:10.1007/s13398-014-0173-7.2.

[17] Pillay A, Prabhakar Karthikeyan S, Kothari DP. Congestion management in power systems - A review. Int J Electr Power Energy Syst 2015;70:83-90. doi:10.1016/j.ijepes.2015.01.022.

[18] Xu B, Abur A. State Estimation of Systems With UPFCs Using the Interior Point Method. IEEE Trans Power Syst 2004;19:1635-41. doi:10.1109/TPWRS.2004.831703.

[19] Rakpenthai C, Premrudeepreechacharn S, Uatrongjit S. Power system with multi-type $\{$ FACTS $\}$ devices states estimation based on predictor-corrector interior point algorithm. Int J Electr Power EnergySyst2009;31:160-6. doi:http://dx.doi.org/10.1016/j.ijepes.2008.10.010.

[20] Kim B-H, Kim H, Moon Y-H. State Estimation of Electric Power Systems with FACTS and HVDC Devices. J Int Counc Electr Eng 2011;1:247-51. doi:10.5370/JICEE.2011.1.3.247.

[21] Chen J, Abur A. Placement of PMUs to Enable Bad Data Detection in State Estimation. IEEE Trans Power Syst 2006;21:160815. doi:10.1109/TPWRS.2006.881149.

[22] Angeles-Camacho EACRF-EHAC. FACTS: Modelling and Simulation in Power Networks. vol. 53. John Wiley \& Sons; 2004. doi:10.1017/CBO9781107415324.004.

[23] Monticelli A, Garcia A. Reliable Bad Data Processing for RealTime State Estimation. IEEE Trans Power Appar Syst 1983;PAS-102:1126-39. doi:10.1109/TPAS.1983.318053.

[24] Abdel-Rahim AMM, Padhy NP. Newton-Raphson UPFC model for power flow solution of practical power networks with sparse techniques. 2004 IEEE Int. Conf. Electr. Util. Deregulation, Restruct. Power Technol. Proc., vol. 1, IEEE; n.d., p. 77-83. doi:10.1109/DRPT.2004.1338472.

[25] Taylor P, Sodhi R, Srivastava SC, Singh SN. Electric Power Components and Systems Phasor-assisted Hybrid State Estimator Phasor-assisted Hybrid State Estimator 2010:37-41. doi:10.1080/15325000903376925. 